\title{
Evaluation of Age-Gene Correlation and the Association with Hypertriglyceridemia Using Adiponectin Receptor Single Nucleotide Polymorphism: A Potential Genetic Screening to Lower Risk of Vascular Disease in Young Asian Males
}

\author{
William C. W. Huang ${ }^{1,2}$, Yi-MeiJoy Lin ${ }^{3}$, Ching Che J. Chiu ${ }^{4}$, Chia-Huei Chiu ${ }^{4} \&$ Fu-Sheng Chang ${ }^{1}$ \\ ${ }^{1}$ Department of Family Medicine, Show-Chwan Memorial Hospital, Changhua, Taiwan ROC \\ ${ }^{2}$ Department of Life Science, National Chung Hsing University, Taichung, Taiwan ROC \\ ${ }^{3}$ Institute of Biomedical Sciences, National Chung Hsing University, Taichung, Taiwan ROC \\ ${ }^{4}$ Department of Research \& Project Management, AHMC Health System, Alhambra, CA, USA \\ Correspondence: Fu-Sheng Chang, Department of Family Medicine, Show-Chwan Memorial Hospital, \\ Changhua, Taiwan ROC. Tel: 1-909-662-2698. E-mail: claire0506@gmail.com
}

Received: March 18, 2017

Accepted: April 5, 2017

Online Published: May 2, 2017

doi:10.5539/hes.v7n2p61

URL: http://doi.org/10.5539/hes.v7n2p61

\begin{abstract}
Purpose: This study was to investigate whether there is an age dependent effect on the association between ADIPPOR1 SNP and hypertriglyceridemia for each gender.

Materials and Methods: 116 individuals aged 20 and above who claimed to be healthy were enrolled and grouped into male and female populations. Blood samples were taken to determine hypertriglyceridemia and genomic variants. Sample t-tests were performed for basic comparison. To ascertain the contribution of genetic variants and age to lipid metabolism, a multivariate logistic regression analysis was conducted to identify the correlates for hypertriglyceridemia adjusting for life styles.
\end{abstract}

Results: For males, individuals with hypertriglyceridemia tended to be younger ( $p$-value $=0.02$ ), less stressed (0.05), and have a higher proportion of ADIPOR1 minor allele carriers (0.03). However, no significant differences were found in age, stress, diet, and genetic variances in females. In regression analysis, males showed age-gene correlation with 1.5 times higher detection of hypertriglyceridemia risk when both factors were considered. In contrast, females showed no correlation between age-gene. In addition, age was positively associated with hypertriglyceridemia in females while males showed an inverse association.

Conclusion: Our findings presented data that suggests age may be a contributing factor to the association between ADIPOR1 and hypertriglyceridemia in males while age showed a significant inverse association with hypertriglyceridemia. Thus, age-gene correlation may be implied during primary practice to encourage lifestyle adjustments by screening for ADIPOR1 SNP minor allele carriers to prevent cerebrovascular disease in males.

Keywords: ADIPOR1 SNP, age-gene interaction, hypertriglyceridemia, male

\section{Introduction}

Vascular disease has contributed billions in health care costs annually in the United States (Go et al., 2013). Lipid levels are important determinants of various vascular diseases, such as hypertriglyceridemia. Hypertriglyceridemia is significantly associated with lacunar infarction (Laloux, Galanti, \& Jamart, 2004) and studies have demonstrated that adiponectin receptor 1 (ADIPOR1) Single Nucleotide Polymorphism (SNP) is highly related to hypertriglyceridemia (Huang, Chen, \& Yang, 2015) and diabetes (Mather et al., 2008). The potential of using SNP as a preventive measure to identify young individuals at high risk of metabolic abnormalities and ultimately, Cerebral Vascular Disease (CVD) appeared worth exploring.

Moreover, gender may impact the ADIPOR1 SNP expression through estrogen levels. Mauro et al. found that estrogen interferes with adiponectin signal transduction via ADIPOR1 in women (Mauro et al., 2014). Furthermore, Tomeick also suggested age-associated estrogen (E (2))-deficiency alters the ER $\alpha / \beta$ ratio in adipose tissue and increases risk for metabolic disease via adiponectin activated mechanisms in female rats 
(Tomicek, Lancaster, \& Korzick, 2011). Although the exact mechanism has not been established in humans, our previous clinical findings demonstrated that ADIPOR1 was significantly associated with hypertriglyceridemia $(\mathrm{OR}>15)$ in men (Huang et al., 2015). In addition, if estrogen and its receptors were age associated, gene expression in gender may also vary by age and investigating age-gene association for each gender specifically may provide valuable findings for future novel bio-molecular studies of metabolic disease. Furthermore, increased lipid levels could be inversely associated with age in specific genes (Dumitrescu et al., 2011), which may be crucial for preventive genome program by assuming that vascular risk is predisposed at a young age and early educational intervention may alter the disease outcome.

Research has also presented that incorporating age-gene interaction into the analysis of lipids can enhance gene discovery and have a greater ability for detections than genome-wide association (Simino et al., 2014). Currently, the calculations of vascular disease risk such as CVD are based on easily accessible biochemical markers and metabolic risks, such as hypertriglyceridemia. Thus, investigating the age dependent effect of the association between ADIPOR1 SNP and hypertriglyceridemia among each gender may have implications for better and earlier detection of CVD risk among healthy individuals. Because we were unaware of any previous studies of this nature, the present study was initiated as a preliminary investigation on hypertriglyceridemia and age-ADIPOR1 correlation by gender.

\section{Method}

The epidemiological investigation was conducted in Taiwan, Republic of China. This study is a part of a nationwide preventive genome program on risks for cardiovascular disease, and the protocol was approved by the Show-Chwan Memorial Hospital IRB board (IRB: \#1020602) in accordance with the principles of the Declaration of Helsinki as revised in 2008.

Study recruited subjects aged 20 and above with no medication history and claimed to be healthy at hospital-affiliated clinics. The life style survey was conducted regarding stress and adverse diet behavior according to the questionnaire provided by the Health Promotion Administration in Taiwan while participants were examined for biochemical abnormality. Stress and adverse diet behavior were scored from 0 to 12 and 0 to 36 points, respectively and biochemical markers such as plasma glucose, TG, cholesterol, high density lipoprotein, low density lipoprotein, etc., were collected from blood sample after an overnight (12-hour) fast.

ADIPOR1 SNP variant (rs1342387) was selected to be significant loci for metabolic disorder. Although located on chromosome 1 intron region (http://www.ncbi.nlm.nih.gov/snp), the gene might interfere with the coding of mRNA (NM_001290553.1). Previous meta-analyses demonstrated that genes located at intron regions might have a higher statistical significance to clinical manifestation than genes at the promotor and exon region (Gao et al., 2013). As of today, there is evidence of disease association of rs 1342387 with hypertriglyceridemia (Huang et al., 2015) that might lead to diabetes (Mather et al., 2008) and stroke (Han et al., 2015).

Genomic DNA was processed and carried out in a laboratory center similar to our previous study methodology using TaqMan SNP Genotyping Assays (Applied Biosystems, Foster City, CA, USA) (Huang et al., 2015). The genotypes of each sample were validated by assessing the restriction fragment length polymorphism in agarose gel electrophoresis. Our study had recruited a total of 116 subjects with 67 females and 49 males. Hardy-Weinberg equilibrium were tested for allele frequency and demonstrated that the alleles may be carried through generations (chi-square test, p-value $>0.05$ ) (Crow, 1999) with homozygous minor allele of 9\%, heterozygous of 52\%, and homozygous major allele of 39\%. According to Gao et al., meta-analysis showed that grouping the SNP base on minor allele frequencies might demonstrate the dominant effect of single allele among the Asian population (Gao et al., 2013). Thus, the population was categorized in two groups based on ADIPOR1 SNP variants, minor allele carrier (T/T, T/C) and the homozygous major allele (C/C). Hypertriglyceridemia was defined based on the National Cholesterol Education Program for metabolic syndrome (TG $\geq 8.33 \mathrm{mmol} / \mathrm{L})$.

Analysis was conducted separately by gender because previous studies had found the ADIPOR1 SNP was expressed differently in males and females. Binary association between hypertriglyceridemia and SNP was assessed using a Fisher exact test while the association with age, stress and diet was assessed using a two sample independent $\mathrm{t}$-test. In order to ascertain the contribution of genetic variants and age to lipid metabolism, a multivariate logistic regression analysis was performed to identify the correlates for hypertriglyceridemia adjusting for life styles. The relative OR for each factor was expressed as exponentiation of the $\beta$ coefficient (Exp (B)) with a p-value. Potential age-gene correlation was demonstrated by removing genetic variables from the regression model and comparing the difference in males and females separately. Statistical analyses were performed using SPSS software, where $p$-values $<0.05$ were considered statistically significant. 


\section{Results}

Table 1 presented the basic characteristics and genetic variance for participants with hypertriglyceridemia (hypertriglyceridemia group) and normal levels of triglyceride (normal group) for males and females separately. Both genders showed differences in the association between age and hypertriglyceridemia. For males, individuals with hypertriglyceridemia tended to be younger ( $p$-value $=0.02$ ), less stressed $(0.05)$, and have a higher proportion of ADIPOR1 minor allele carriers (0.03). However, no significant differences were found in age, stress, diet, and genetic variances in female.

Tables 2-4 presented the results of the following multivariate logistic regression models adjusting stress and diet for males and females separately: 1) ADIPOR1 only, 2) ADIPOR1 and age, and 3) age only. For male participants, when age was not included in the model, ADIPOR1 minor allele carriers showed significant association with hypertriglyceridemia with OR of 16.3 (0.02). With the addition of age in the model, ADIPOR1 showed a 1.5 fold increase in the strength of association with hypertriglyceridemia with an OR of $23.6(0.02)$. In addition, age also showed a significant negative correlation with hypertriglyceridemia in male (0.04). In contrast, only age showed a significant positive correlation with hypertriglyceridemia in females, and when the genetic factor was removed from the equation, age remained a significant determinant for hypertriglyceridemia (0.04). However, in males, age was rendered insignificant. The results suggested that the association between ADIPOR 1 and hyperglyceridemia in each gender might differ by age.

Table 1. Basic characteristics and genetic variants between normal and hypertriglyceridemia of the different gender population

\begin{tabular}{|c|c|c|c|c|c|c|c|c|}
\hline & \multicolumn{4}{|c|}{ Male } & \multicolumn{4}{|c|}{ Female } \\
\hline & $\begin{array}{c}\text { Normal } \\
(\mathrm{Mean} \pm \mathrm{SD})\end{array}$ & & $\begin{array}{l}\text { Abnormal } \\
(\text { Mean } \pm \text { SD) }\end{array}$ & $P$ & $\begin{array}{c}\text { Normal } \\
(\mathrm{Mean} \pm \mathrm{SD})\end{array}$ & & $\begin{array}{l}\text { Abnormal } \\
(\text { Mean } \pm \text { SD) }\end{array}$ & $P$ \\
\hline Age & $48.2 \pm 13.9$ & & $43.3 \pm 9.2$ & 0.02 & $44.3 \pm 10.5$ & & $51 \pm 14.2$ & 0.063 \\
\hline Diet & $11.18 \pm 6.40$ & & $11.38 \pm 4.03$ & 0.29 & $10.27 \pm 5.71$ & & $9.5 \pm 5.71$ & 0.81 \\
\hline \multirow[t]{2}{*}{ Stress } & $2.82 \pm 2.53$ & & $1.92 \pm 2.06$ & 0.05 & $4.85 \pm 3.05$ & & $4.42 \pm 2.50$ & 0.31 \\
\hline & $\mathrm{n}$ & $\%$ & $\mathrm{n}$ & $\%$ & $\mathrm{n}$ & $\%$ & $\mathrm{n}$ & $\%$ \\
\hline \multicolumn{9}{|l|}{ ADIPOR1 } \\
\hline$(\mathrm{M} / \mathrm{M})$ & 16 & $47 \%$ & 2 & $13 \%$ & 22 & $41 \%$ & 4 & $31 \%$ \\
\hline$(\mathrm{mM} / \mathrm{mm})$ & 18 & $53 \%$ & 13 & $87 \%$ & 32 & $59 \%$ & 9 & $69 \%$ \\
\hline $\begin{array}{l}\text { Fisher test } \\
\text { (2 sided) }\end{array}$ & \multicolumn{4}{|c|}{$P$ value $=0.03$} & \multicolumn{4}{|c|}{$P$ value $=0.75$} \\
\hline
\end{tabular}

Note. ${ }^{*}$ Statistical significance $\mathrm{p}<0.05$.

M/M: Homozygous major allele

$\mathrm{mM} / \mathrm{mm}$ : Minor allele carrier

Normal TG: $\mathrm{TG}<8.33 \mathrm{mmol} / \mathrm{L}$

Abnormal TG: TG $\geq 8.33 \mathrm{mmol} /$ 
Table 2. Multivariate logistic regression using TG $\geq 8.33 \mathrm{mmol} / \mathrm{L}$ as abnormal level including only genetic factor

\begin{tabular}{lcccccc}
\hline & \multicolumn{3}{c}{ Male } & \multicolumn{3}{c}{ Female } \\
\cline { 2 - 7 } & Exp (B) & Wald & $p$-value & Exp (ß) & Wald & $p$-value \\
\hline ADIPOR1 & 12.81 & 5.13 & $0.02^{*}$ & 2.36 & 1.38 & 0.24 \\
Stress & 0.77 & 2.46 & 0.12 & 0.98 & 0.04 & 0.84 \\
Diet & 1.03 & 0.25 & 0.62 & 0.96 & 0.39 & 0.53 \\
\hline
\end{tabular}

Note. * Statistical significance $\mathrm{p}<0.05$.

Table 3. Multivariate logistic regression of age effect by including age in the model

\begin{tabular}{lcccccc}
\hline & \multicolumn{3}{c}{ Male } & \multicolumn{3}{c}{ Female } \\
\cline { 2 - 6 } & Exp (ß) & Wald & $p$-value & Exp $(\beta)$ & Wald & $p$-value \\
\hline ADIPOR1 & 21.5 & 5.74 & $0.02^{*}$ & 2.7 & 1.68 & 0.2 \\
Age & 0.9 & 4.05 & $0.04^{*}$ & 1.1 & 5.1 & $0.02^{*}$ \\
Stress & 0.7 & 4.25 & $0.04^{*}$ & 0.93 & 0.34 & 0.56 \\
Diet & 1.0 & 0.03 & 0.86 & 1.0 & 0.01 & 0.94 \\
\hline
\end{tabular}

Note. ${ }^{*}$ Statistical significance $\mathrm{p}<0.05$.

Table 4. Multivariate logistic regression of gene effect by removing ADIPOR1 from the model

\begin{tabular}{ccccccc}
\hline & \multicolumn{3}{c}{ Male } & \multicolumn{3}{c}{ Female } \\
\cline { 2 - 7 } & Exp (ß) & Wald & $p$-value & Exp (ß) & Wald & $p$-value \\
\hline Age & 1.0 & 2.64 & 0.10 & 1.1 & 4.45 & $0.04^{*}$ \\
Stress & 0.8 & 1.87 & 0.17 & 0.91 & 0.63 & 0.43 \\
Diet & 1.0 & 0.08 & 0.78 & 1.03 & 0.26 & 0.61 \\
\hline
\end{tabular}

Note. ${ }^{*}$ Statistical significance $\mathrm{p}<0.05$.

\section{Discussion}

Our study presented the following findings: (a) ADIPOR1-age correlation was crucial for hypertriglyceridemia in males, (b) age was inversely associated with hypertriglyceridemia in males, and (c) age was positively correlated to hypertriglyceridemia in females despite genetic variance.

This suggested that gene-age correlation is important while performing regression analysis of age and abnormal lipid level association in traditional epidemiologic studies. Epidemiologic studies have conventionally taken gender into account (Lancet, 2011). However, there was not much attention to address to the genetic and age influence upon metabolic risk in different genders. Given the studies regarding difference in TG metabolism between men and women (Olefsky, Farquhar, \& Reaven, 1974), our studies further highlighted the possibility of using ADIPOR1 SNP variant (rs1342387) to survey hypertriglyceridemia and that taking gender into account could obtain a much higher risk ratio $(\operatorname{Exp}(\beta)=16.31)$ (Table 2). By incorporating age-gene correlation into risk assessments, the odds ratio would increase by $150 \%$ (Exp (ß)-24), more so than considering genders alone (Table 3). Like previous research had suggested (Simino et al., 2014), our research showed increased detection of genetic variance (1.5 times more) in males by adding the age-gene correlation into the study. Furthermore, genetic and age variable together also significantly affect the correlation between stress and hypertriglyceridemia in males. Without the age (Table 2) or genetic variable (Table 4), stress showed no significance using the logistic model. By showing the correlation between age, gene and life style in this study was particularly profound and deserves future attention. 
The age-gene correlation in hypertriglyceridemia was only demonstrated among the male population. Neglecting genetic variables did not alter the significance of age contribution to female hypertriglyceridemia. Age in females was positively correlated to hypertriglyceridemia, with or without genetic variance (Tables $3 \& 4$ ). The disparity demonstrated that the mechanism of age-gene correlation in triglyceride metabolism was gender specific and might be the result of interaction between estrogen, estrogen receptors, adiponectin and adiponectin receptors. These clinical findings coincided with Tomicek's animal study which had suggested that age-associated alteration in ER paired with decreased PPAR- $\gamma$ levels might predispose E (2)-deficient postmenopausal women for increased adiposity and associated metabolic and cardiovascular disease risk and reduced circulating adiponectin and adiponectin receptors levels might contribute to age and E (2)-deficiency linked disease progression (Tomicek et al., 2011). Levels of estrogen and adiponectin together with interaction between ADIPOR and ER may alter the secondary messenger and thus result in significant age-ADIPOR correlation of triglyceride metabolism.

With known physiology, one may anticipate young males to be the most E (2)-deficient group and those ADIPOR1 minor allele carriers are prone to impaired adiponectin receptor function. Together, the alteration in ADIPOR1 SNP and a lack of estrogen may be linked to metabolic disease progression and significant expression of hypertriglyceridemia in young males when compared to elder males or females. By presenting the significant inverse association of age and hypertriglyceridemia $(\mathrm{OR}=0.9$ and $\mathrm{p}$-value $=0.04)$, our study had demonstrated that genomic variants may be applied in early prevention of metabolic abnormalities in specific genders during primary practice. Our findings may suggest the primary practice to use ADIPOR1 SNP (rs1342387) to screen juvenile males with a family history of vascular disease so that lifestyle adjustments such as stress exercise can be employed upon minor allele carriers to alter vascular disease progression.

However, the future implications of our study have three limitations. First, this study was only conducted within the Asian population and may not be applied to other ethnicities. Second, the sample population was small due to various limitations and thus should be incorporated into major genetic preventive program studies for better statistical results. Third, we evaluated only the association with metabolic risk, not disease outcomes, thus should be implied with further studies.

In conclusion, first the study provided initial clinical evidence for future bio-molecular studies regarding the age-gene correlation through ADIPOR and ER in lipid metabolism. Second, disease is often defined and risk calculated by various biochemical abnormalities. Hypertriglyceridemia is a significant risk factor for both ischemic stroke (Laloux et al., 2004) and coronary disease (Sarwar et al., 2007). By incorporating genetic variance into the calculation of disease risk, one may obtain a more efficient risk assessment model in the future. Third, lipid levels were shown with age variance (Snieder, van Doornen, \& Boomsma, 1997) and exhibited correlation among relatives with similar age (Iselius, 1979). Our findings suggested future clinical implication of providing genetic risk assessment for siblings of young individuals and descendants of elder individuals with metabolic disease and intervening with group counseling and life style adjustments for those who carry high risk alleles. If the above statements may be carried out in the near future, this study approach seems worthy of further evaluation in other races and in larger populations.

\section{Acknowledgements}

Professor KD Yang's research team had supported the biomolecular test of the study. This paper was editorially assisted by Show-Chwan Memorial Hospital Research Assistant Center and Erica Hsu.

\section{References}

Crow, J. F. (1999). Hardy, Weinberg and language impediments. Genetics, 152, 821-825.

Dumitrescu, L., Brown-Gentry, K., Goodloe, R., Glenn, K., Yang, W., Kornegay, N., ... Crawford, D. C. (2011). Evidence for age as a modifier of genetic associations for lipid levels. Annals of Human Genetics, 75, 589-597. https://doi.org/10.1111/j.1469-1809.2011.00664.x

Gao, M., Ding, D., Huang, J., Qu, Y., Wang, Y., \& Huang, Q. (2013). Association of genetic variants in the adiponectin gene with metabolic syndrome: A case-control study and a systematic meta-analysis in the Chinese population. PLoS One, 8, e58412. https://doi.org/10.1371/journal.pone.0058412

Go, A. S., Mozaffarian, D., Roger, V. L., Benjamin, E. J., Berry, J. D., Borden, W. B., ... Turner, M. B. (2013). Heart disease and stroke statistics-2013 update: A report from the American Heart Association. Circulation, 127, e6-e245. https://doi.org/10.1161/CIR.0b013e318282ab8f 
Han, Q., Shu, Z., Liang, X., Mi, R., Yang, L., \& Li, P. (2015). Relationship between adiponectin receptor 1 gene polymorphisms and ischemic stroke. International Journal of Clinical and Experimental Medicine, 8, 16719-16723.

Huang, W. C. W., Chen, R. F., \& Yang, Y. Y. (2015). Adiponectin Receptor 1 Single Nucleotide Polymorphism Is Highly Associated with Hypertriglyceridemia in Asian Male-A Novel Genetic Screening to Reduce Risk of Cerebrovascular Disease. World Journal of Neuroscience, 5, 323-327. https://doi.org/10.4236/wjns.2015.55029

International Physical Activity Questionnaire. (2013). Health Promotion Administration, Ministry of Health and Welfare. Retrieved

http://www.hpa.gov.tw/BHPNet/Web/Easy/FormCenterShow.aspx?No=201201130001

Iselius, L. (1979). Analysis of family resemblance for lipids and lipoproteins. Clinical Genetics, 15, 300-306. https://doi.org/10.1111/j.1399-0004.1979.tb01738.x

Laloux, P., Galanti, L., \& Jamart, J. (2004). Lipids in ischemic stroke subtypes. Acta Neurologica Belgica, 104, 13-19.

Lancet. (2011). Taking sex into account in medicine. Lancet, $378,1826$. https://doi.org/10.1016/S0140-6736(11)61795-9

Mather, K. J., Funahashi, T., Matsuzawa, Y., Edelstein, S., Bray, G. A., Kahn, S. E., ... Goldberg, R. (2008). Adiponectin, change in adiponectin, and progression to diabetes in the Diabetes Prevention Program. Diabetes, 57, 980-986. https://doi.org/10.2337/db07-1419

Mauro, L., Pellegrino, M., De Amicis, F., Ricchio, E., Giordano, F., Rizza, P., ... Andò, S. (2014). Evidences that estrogen receptor $\alpha$ interferes with adiponectin effects on breast cancer cell growth. Cell Cycle, 13, 553-564. https://doi.org/10.4161/cc.27455

Olefsky, J., Farquhar, J. W., \& Reaven, G. M. (1974). Sex difference in the kinetics of triglyceride metabolism in normal and hypertriglyceridaemic human subjects. European Journal of Clinical Investigation, 4, 121-127. https://doi.org/10.1111/j.1365-2362.1974.tb02324.x

Pressure and diet Questionnaire. (2013). Health Promotion Administration, Ministry of Health and Welfare. Retrieved from http://health99.hpa.gov.tw/OnlinkHealth/OnlinkHealth_list.aspx

Pressure and diet Questionnaire. (n.d.). Health Promotion Administration, Ministry of Health and Welfare, Taiwan. Retrieved July 7, 2013, from http://health99.hpa.gov.tw/OnlinkHealth/OnlinkHealth_list.aspx

Sarwar, N., Danesh, J., Eiriksdottir, G., Sigurdsson, G., Wareham, N., Bingham, S., ... Gudnason, V. (2007). Triglycerides and the risk of coronary heart disease: 10,158 incident cases among 262,525 participants in 29 $\begin{array}{lllll}\text { Western } & \text { prospective } & \text { 4irculation, } & \text { 115, }\end{array}$ https://doi.org/10.1161/CIRCULATIONAHA.106.637793

Simino, J., Kume, R., Kraja, A. T., Turner, S. T., Hanis, C. L., Sheu, W. H., ... Rao, D. C. (2014). Linkage analysis incorporating gene-age interactions identifies seven novel lipid loci: The Family Blood Pressure Program. Atherosclerosis, 235, 84-93. https://doi.org/10.1016/j.atherosclerosis.2014.04.008

Snieder, H., van Doornen, L. J., \& Boomsma, D. I. (1997). The age dependency of gene expression for plasma lipids, lipoproteins, and apolipoproteins. American Journal of Human Genetics, 60, 638-650.

Tomicek, N. J., Lancaster, T. S., \& Korzick, D. H. (2011). Increased estrogen receptor $\beta$ in adipose tissue is associated with increased intracellular and reduced circulating adiponectin protein levels in aged female rats. Gender Medicine, 8, 325-333. https://doi.org/10.1016/j.genm.2011.05.010

\section{Copyrights}

Copyright for this article is retained by the author(s), with first publication rights granted to the journal.

This is an open-access article distributed under the terms and conditions of the Creative Commons Attribution license (http://creativecommons.org/licenses/by/4.0/). 